\title{
CONTRIBLTTI ALLA TEORIA DELLE SESSE IN SISTEMI COMPOSTI DI BACINI LACUSTRI ( $\left.{ }^{*}\right)$
}

\author{
Gerhard Neumann
}

Vi sono in natura laghi e baie marine, che si diramano in uno o più punti o sono collegati con altri bacini marini per mezzo di canali stretti.

Tali laghi e baie marine composti di singoli bacini, come hanno dimostrato osservazioni fatte su modelli e laghi naturali, hanno forme di oscillazione del tutto particolari e spesso complicatissime (sesse). Si sviluppano in essi non solo oscilla. zioni parziali delle parti lacustri delimitate, ma si possono comprovare anche oscillazioni del sistema complessivo, che alla loro volta dipendono dai periodi e dalle dimensioni delle parti separate del lago, e dal modo e dalla grandezza del collegamento di queste parti.

Il tentativo di applicare a tali sistemi composti di bacini lacustri i metodi teorici finora conosciuti per la determinazione dei periodi propri, s'imbatte spesso in difficoltà. Ciò perché le teorie sopra dette partono dalla premessa che il procedimento di oscillazione possa essere descritto in tutto il campo di oscillazione dalla stessa funzione stabile di tempo e luogo.

Nel caso di forti restringimenti trasversali e di canali stretti che collegano tra loro bacini lacustri piuttosto grandi, questa premessa tuttavia non si avvcra. Si devono piuttosto interpretare le singole parti del sistema lacustre come campi separati di oscillazione, che si influenzano vicendevolmente e per effetto della loro azione simultanea (accoppiamenti) possono comparire anche oscillazioni libere del sistema complessivo. Se p. es. un lago $I$, di qualsiasi configurazione esso sia, si trova collegato ad una estremità mediante uno stretto canale II con un secondo bacino d'acqua III, come rappresenta schematicamente la fig. 1, allora sono anzitutto possibili di per sé in ogni bacino lacustre oscillazioni libere. Ora però, ad ogni crescita dello specchio del lago una certa quantità defluirà dall'estremità chiusa in modo incompleto dal canale di collegamento nella vicina parte del lago, e abbassandosi lo specchio del lago, nuovamente rifluirà. Per questo non solo si muta il periodo proprio dei singoli bacini parziali in modo determinato, ma appare anche una oscillazione composta del sistema complessivo. Per mezzo della massa d'acqua fluente e rifluente nel canale di collegamento i due bacini parziali " sono accoppiati » tra loro. Si tratta ora di determinare accanto alle oscillazioni parziali dei singoli laghi, anche i periodi del sistema complessivo accoppiato. Appartiene a questo problema tutta una serie di altri casi di complicate combinazioni di bacini, che sono importanti nella teoria delle sesse. Un ruolo speciale ha la questione, come possano p. es. mutarsi i periodi propri di un lago, quando il lago sia collegato col mare libero mediante aperture laterali o canali. Nella fig. 2 è rappresentato schematicamente un tale sistema di bacino lacustre aperto lateralmente, per chiarire la posizione del problema.

Per problemi di oscillazione acustica di simile natura e in circuiti oscillanti

(*) Traduzione in lingua italiana della Nota di pag. 15. 
elettromagnetici, si è introdotto con successo il concetto di impedenza per la determinazione dei periodi propri di tali sistemi.

L'autore (1), muovendo dall'esempio di un punto oscillante di massa, ha trasferito questo concetto di impedenza a sistemi continui, e lo ha applicito concettualmente alla teoria delle sesse. Venne quindi definito come impedenza idrodinamica nelle oscillazioni di masse d'acqua il rapporto:

$$
Z=\frac{p_{0}}{S(\partial c / \partial t)_{\max }}-\frac{\text { ampiezza di pressione }}{\text { superficie } \times \text { ampiezza di velocità }}
$$

Per singole formazioni caratteristiche di oscillazioni questa grandezza è da ricavare dalle equazioni idrodinamiche del movimento. In sistemi composti di bacini lacustri, le impedenze $Z_{1}, Z_{3}, Z_{3}, \ldots$ dei membri parziali del sistema si devono addizionare, come p. es. le resistenze nei circuiti elettromagnetici oscillanti. Se l'impedenza di un sistema di oscillazione è nota, allora le frequenze proprie $\omega_{v}=\frac{2 \pi}{T_{v}}$ del sistema si ricavano dalla condizione $Z=0$, trascurando l'attrito (altrimenti $Z=$ Min.).

Per l'impedenza di sistemi semplici di oscillazione e dei loro componenti ven. nero dedotte le formule (1), che permettono di calcolare il periodo proprio delle sesse in combinazioni di bacini lacustri di qualsivoglia conformazione, considerando con soddisfacente precisione la figura irregolare del bacino. Esse completano le note teorie delle sesse, perché possono essere applicate lá, dove queste ultime non sono sufficienti, quindi p. es. per i laghi con forti restringimenti di bacini, per combinazioni di bacini lacustri, per masse d'acqua incompletamente delimitate e cosi via.

Per i termini più importanti di un sistema oscillante le grandezze $Z$ sono indicate qui sotto. Per la loro deduzione si rimanda alla trattazione originale (1):

1) Per bacini chiusi da un solo lato dalla superficie trasversale media $S=b h$ $(b=$ larghezza, $h=$ profondita) vale

$$
Z=-\frac{i \cap c}{S} \operatorname{cotg} \frac{\omega L}{c} \quad, \quad L=\lambda / 4
$$

dove $i=\sqrt{-1}, c=\sqrt{g h}, \omega=\frac{i \pi}{T} L=$ lunghezza del bacino, $\lambda=$ lunghezza d'onda e $\varrho$ significa la densità dell'acqua. La stessa formula vale anche per un bacino chiuso da ambo le parti, solo che in questo caso $L$ è da porsi $L=\lambda / 2$. mità è :

2) L'impedenza di un bacino d'acqua esteso aperto ad entrambe le estre-

$$
Z=\frac{i_{\varrho} c}{S}, \operatorname{tang} \frac{\omega L}{c} \quad, \quad L=\lambda / 2
$$

3) Per una apertura stretta del flusso, o per un canale lungo dalla sezione trasversale $q=b h$ e di lunghezza $L$ vale:

$$
Z_{\mathrm{q}}=\frac{i \varrho \omega L}{q}
$$


Per la lunghezza geometrica $L$ del canale di deflusso si deve eventualmente introdurre una grandezza "effettiva " $L^{\prime}=L+\alpha$. Con il tratto aggiunto $\alpha$ si considera la massa d'acqua insieme oscillante in prossimità dellapertura.

Si tratta qui dell'apporto di una specie di "correzione di fase » sulla quale l'Autore (5) ha fatto speciali ricerche e sulle quali riferirà a parte.

4) Un bacino d'acqua collegato mediante una stretta apertura col mare aperto o con un altro bacino maggiore, ha una forma particolarmente preferita di oscillazione se le dimensioni di questo bacino sono presso a poco uguali in tutte le direzioni (forma circolare). Vi possono essere oscillazioni, nelle quali tutto il livello d'acqua del lago sale e s'abbassa uniformemente.

A questa forma di oscillazione corrisponde acusticamente il risonatore di Hemoltz. Il calcolo per l'impedenza di un tale sistema da:

$$
Z=\frac{i \omega \omega L}{q}-\frac{i \varrho c^{2}}{Q \omega}
$$

dove $Q$ indica il volume del bacino d'acqua. $Z$ si compone qui di due parti, della impedenza dell'apertura di deflusso $Z q$ (formula 4), e di un secondo membro $Z=-i \varrho c-Q(1)$ impedenza del volume dacqua delimitato. Con $Z=0$ deriva da [5]

$$
-\frac{g}{F \omega} \div \frac{m I}{q}=0 \quad \text { e } \quad T=2 \pi / / \frac{F L}{g \varphi}
$$

( $F=$ area della superficie del lago). Questa è una equazione di periodo nota nella teoria delle sesse e specificatamente ottenuta dai giapponesi; tale equazione segue qui immediatamente quale risultato secondario dalla teoria più generale dell'impedenza. Casi specialissimi furono trattati anche da $\mathrm{N}$. Zeilon (6), che ha calcolato i periodi delle sesse in sistemi ramificati di bacini lacustri, derivandoli da equa. zioni idrodinamiche di movimento e considerando le condizioni limite. Queste ri. cerche anteriori sono qui ricordate solo perché esse sono tutte contenute nel metodo proposto sotto un punto di vista unitario. Le formule ricavate da Zeilon si possono facilmente scrivere con l'aiuto della teoria dell’impedenza come casi speciali :

a) Lago chiuso ramificato (fig. 3 ).

Con questo esempio si può subito spiegare il metodo.

Il Iago I si biforca nelle ramificazioni II e III. Oscillando l'acqua può fluire tanto nel bacino II quanto nel bacino III. I bacini II e III si trovano quindi "inseriti parallelamente" con le impedenze $Z$. e $Z$ a dietro al bacino $I$ con impedenza $Z_{1}$. Se indichiamo con $P$ l'impedenza complessiva di II e III, risulta:

$$
\frac{1}{P}=\frac{1}{Z_{2}}+\frac{1}{Z_{3}} \quad \text { e } \quad P=\frac{Z_{2} Z_{3}}{Z_{2}+Z_{3}} .
$$

I bacini I e (II + III) sono però " uniti in serie », cioè

$$
Z_{1}+P=Z_{1}+\frac{Z_{2} Z_{3}}{Z_{2}+Z_{3}}
$$


La condizione per la frequenza propria diventa allora

e con la [2]

$$
\frac{1}{Z_{1}}+\frac{1}{Z_{2}}+\frac{1}{Z_{3}}=0
$$

$$
\underset{-c_{1}}{b_{1}} \frac{h_{1}}{c_{1}} \operatorname{tag} \frac{\omega L_{1}}{c_{1}}+\frac{b_{2} h_{2}}{c_{2}} \operatorname{tag} \frac{\omega L_{2}}{c_{2}}+\frac{b_{3} h_{3}}{\varrho c_{3}} \operatorname{tag} \frac{\omega L_{3}}{c_{3}}=0 .
$$

Moltiplicando per $g$ (accelerazione di gravità), otteniamo

$$
b_{1} c_{1} \operatorname{tag} \frac{\omega L_{1}}{c_{1}}+b_{2} c_{2} \operatorname{tag} \frac{(1) I_{2}}{c_{2}}+b_{3} c_{3} \operatorname{tag} \frac{\omega L_{3}}{c_{3}}=0,
$$

cioè l'equazione di Zeilon (6).

Simili equazioni trascendenti si possono dedurre anche per il caso che uno dei laghi, due, o tutti e tre siano aperti all'estremità. Se p. es. sono aperti all'estremità i laghi I e II, ma il lago III è chiuso, ne deriva

$$
-b_{1} c_{1} \operatorname{cotg} \frac{\omega L_{1}}{c_{1}}-b_{2} c_{2} \operatorname{cotg} \frac{\omega L_{2}}{c_{2}}+b_{3} c_{3} \operatorname{tg} \frac{\omega L_{2}}{c_{3}}=0 .
$$

Analogamente si possono porre equazioni per lo più trascendenti, per la determinazione di (1) e $T$ anche per qualsivoglia altre combinazioni di bacini lacustri, come l'Autore ha dimostrato con una serie di esempi.

Il metodo non si limita tuttavia a bacini lacustri, la cui larghezza e profondita sono costanti. Abbiamo scelto qui nelle figure schematiche solo tali casi semplici per raffigurare più evidentemente il problema. Il metodo proposto si può applicare, come l'autore ha dimostrato con numerosi esempi di laghi alpini dalla conformazione complicata, ad ogni lago naturale dalla configurazione irregolare. Aggiungiamo qui per chiarimento alcuni esempi :

1) Il Königssee nella Baviera Superiore viene diviso da un restringimento del bacino presso $\mathrm{S}$. Bartolomeo in due bacini parziali di ineguale grandezza.

Vi si può applicare la fig. l schematica. Il sistema accoppiato complessivo consta qui di due laghi parziali I e II, collegati dai un canale II. Se indichiamo limpedenza dei membri secondo la serie con $Z_{l}, Z_{4}, Z_{3}$ (fig. 1), allora con le formule [2] e [4] la condizione per la frequenza propria è:

$$
\text { . } Z_{1}+Z_{\mathrm{q}}+Z_{3}=-\frac{c_{1}}{\hat{D}_{1}} \operatorname{cotg} \frac{\omega L_{1}}{c_{1}}+\frac{\omega L}{q}-\frac{c_{3}}{S_{3}} \operatorname{cotg} \frac{\omega L_{3}}{c_{3}}=0
$$

$$
\omega=\frac{2 \pi}{T}=\frac{q}{L}\left(\frac{c_{1}}{S_{1}} \operatorname{cotg} \frac{\omega L_{1}}{c_{1}}-\frac{c_{*}}{S_{1}} \operatorname{cotg} \frac{\omega L_{s}}{c_{3}}\right) \text {. }
$$

Perciò possiamo anche scrivere

$$
\frac{2 \pi}{T}=\frac{q}{L}\left(\frac{c_{1}}{S_{1}} \operatorname{cotg} \pi \frac{T \mathrm{l}}{T}+\frac{c_{3}}{S_{3}} \operatorname{cotg} \pi \frac{T_{\mathrm{LIT}}}{T}\right)
$$


Nell'equazione stanno ora in luogo di $2 L_{1} / c_{1}$, e $2 L_{3} / c_{3}$ i periodi Tr e TIII delle oscillazioni parziali dei singoli laghi, come apparirebbero nel canale di collegamento pensando la chiusura completa. Possono essere determinate considerando ulteriormente la configurazione del bacino, secondo il metodo dei resti di Defant. II periodo che appare nell'equazione [6] è il periodo proprio del sistema complessivo accoppiato. Con I e III sono determinati anche i valori effettivi $c_{1}$ e $c_{s}$, così che $T$ si può calcolare dalla equazione trascendente [6]. Per il periodo principale di tutto il Konigssee risulta $T=11,0$ Min. Da registrazioni limnografiche A. EnAros (8) ha determinato il periodo principale in 11,6 Min. Le oscillazioni erano però fortemente smorzate, così che il periodo calcolato, tenendo conto dello smorzamento si alzerà alquanto.

2) Particolarmente notevoli sono le sesse sul Waginger-Tachingersee, che, come dice il nome, è un lago doppio. Fra i due laghi parziali c'è uno stretto collegamento presso Tettenhausen. La lunghezza del canale di collegamento è circa $140 \mathrm{~m}$, con una sezione trasversale del canale di soli $90 \mathrm{~m}$ ".

Per il periodo base delle oscillazioni parziali in entrambi $i$ singoli bacini il metodo di Defant dà $18,0 \mathrm{~min}$. per Wagingersee -e $13,8 \mathrm{~min}$. per il Tachingersee.

La soluzione dell'equazione [6] però dà per il sistema composto WagingerseeTachingersee un periodo per la sessa principale ben maggiore, precisamente $T=64$ min. Tali lente oscillazioni del sistema complessivo sono state effettivamente osservate da Endrös (9), accanto a oscillazioni parziali più brevi dei singoli bacini, per quanto non gli fosse possibile di interpretare teoreticamente questo periodo rilevantemente lungo. Nelle registrazioni limnografiche questa oscillazione appare chiaramente con un periodo medio $T=62 \mathrm{~min}$. Questa sessa si spiega dunque come oscillazione principale del sistema accoppiato Waginger-Tachingersee. Tale risultato, e la sua coincidenza con le osservazioni, dimostrano la praticità del metodo qui applicato. Per convalidare il metodo l'autore (3) ha osservato ancora tutta una serie di altre combinazioni di bacini lacustri di complessa configurazione, che qui ıon possono essere singolarmente esaminati. Interessante è ancora l'esempio del

3) Plan-Heilerwangersee. Il collegamento fra il Plansee, maggiore, col Heiterwangersee, minore, è estremamente angusto, per cui il piccolo Heiterwangersee si deve considerare più che come "bacino di deflusso" per il Plansee, che è più grande. L'equazione del periodo per il sistema lacustre composto diviene in questo caso

$$
\operatorname{cotg} \pi \frac{T_{\mathrm{I}}}{T}=\frac{2 \pi S_{1} I}{c_{1} q} \frac{1}{T}\left(1-\frac{T^{2}}{T_{\mathrm{LI}^{2}}}\right),
$$

dove gli indici 1 si riferiscono al Plansee. $T_{1}$ è il periodo parziale del Plansee e $T_{2}$ quello del Heiterwangersee. $L$ e $q$ sono la lunghezza e la superficie trasversale media del canale collegante. Il periodo $T$ delle sesse nel sistema composto, per la oscillazione fondamentale

$$
T=124 \min
$$

secondo l'equazione [7] è rilevantemente grande. Anche questo lungo periodo di sesse è stato osservato da A. Endros (10), ma fino ad ora poté difficilmente venir spiegato teoreticamente, perchè la durata della oscillazione principale del solo Plansee ammonterebbe a $10 \mathrm{~min}$. Endros supponeva già una «oscillazione di compen- 
so » tra i due lighi. Essendo giusta questa supposizione, il risultato sopra esposto conferma la teoria della impedenza.

E appena necessario osservare, che dalle soluzioni delle equazioni trascendenti dei periodi, che si possano facilmente porre per ogni combinazione di bacini lacustri con l'aiuto del concetto di impedenza, si possono calcolare anche i periodi delle oscillazioni superiori. Non occorre però addentrarsi qui ulteriormente in questa breve relazione.

Una parte speciale spetta agli sbocchi ad una estremità o lateralmente di laghi molto estesi che conducono in un campo marino aperto (fig. 2).

Il periodico defluire e affluire d'acqua attraverso l'apertura muta in determiminato modo i periodi propri delle sesse del lago. La teoria della impedenza mostra inoltre, che a seconda della posizione dell'apertura di deflusso rispetto ai nodi e ai ventri di oscillazione della sessa considerata nel lago, subentra un abbassamento più o meno grande del periodo. Sull'esempio del Frischen Haff, sulla costa Irussiana orientale, collegato col mar Baltico mediante il "Pillaner Tief», si poté mostrare quanto forte è l'influsso di tali aperture sul fianco di masse d'acqua oscillanti. Senza considerare il collegamento laterale con il mar Baltico, il periodo principale delle sesse del Frischen Haff comporterebbe ore 9,7. La teoria qui esposta dà però, considerando il "Pillauer Tief ", un periodo principale di $T=8,05$ ore soltanto, la qual cosa coincide bene con il periodo osservato della sessa di 8 ore.

Questi bacini lacustri aperti lateralmente o a una estremità formano una cosa intermedia tra il "lago " e la "baia del mare ». Essi hanno le loro specialissime sesse. Le questioni inerenti a tali bacini, particolarmente anche la questione della “correzione di bocca » sono trattate in un lavoro speciale.

\section{RIASSUNTO}

L'uso del principio dellimpedenza nella teoria delle sesse conduce ad un metodo semplice e generale per il calcolo dei periodi propri di sistemi lacustri, costituiti da parti singole capaci di oscillazioni proprie. Viene p. es. provato che le equazioni dei periodi dedotte con altri metodi sono facilmente ricondotte a casi particolari della "Impedanztheorie der Seiches》. Inoltre vengono risolti alcuni casi di bacini complicati, rimasti finora insoluti. Nel presente lavoro si riassumono lavori precedentemente condotti dall'Autore e si indica come p. es. il periodo proprio di un lago, unito mediante uno stretto canale ad altro bacino, venga infuenzato dalla diramazione. Il metodo è applicabile non solo ai bacini di larghezzı e profonditì costante, ma anche a bacini di forma irregolare, quali possono essere $i$ laghi alpini. 